OPEN ACCESS

Edited by:

Suhelen Egan,

University of New South Wales,

Australia

Reviewed by:

Irene Wagner-Doebler,

Helmholtz Centre for Infection

Research (HZ), Germany

Paolo Puccetti,

University of Perugia, Italy

*Correspondence:

Robert Krause

robert.krause@medunigraz.at

Specialty section:

This article was submitted to

Microbial Symbioses,

a section of the journal

Frontiers in Microbiology

Received: 21 July 2016 Accepted: 23 December 2016

Published: 10 January 2017

Citation

Krause R, Moissl-Eichinger $C$, Halwachs B, Gorkiewicz G, Berg G, Valentin T, Prattes J, Högenauer $C$ and Zollner-Schwetz I (2017) Mycobiome in the Lower Respiratory Tract - A Clinical Perspective.

Front. Microbiol. 7:2169.

doi: 10.3389/fmicb.2016.02169

\section{Mycobiome in the Lower Respiratory Tract - A Clinical Perspective}

\author{
Robert Krause ${ }^{1 *}$, Christine Moissl-Eichinger ${ }^{1,2}$, Bettina Halwachs ${ }^{3}$, Gregor Gorkiewicz ${ }^{2,3}$, \\ Gabriele Berg ${ }^{4}$, Thomas Valentin ${ }^{1}$, Jürgen Prattes ${ }^{1}$, Christoph Högenauer ${ }^{5}$ and \\ Ines Zollner-Schwetz ${ }^{1}$
}

${ }^{1}$ Section of Infectious Diseases and Tropical Medicine, Department of Internal Medicine, Medical University of Graz, Graz, Austria, ${ }^{2}$ BioTechMed, Medical University of Graz, Graz, Austria, ${ }^{3}$ Institute of Pathology, Medical University of Graz, Graz, Austria, ${ }^{4}$ Institute of Environmental Biotechnology, Graz University of Technology, Graz, Austria, ${ }^{5}$ Division of Gastroenterology and Hepatology, Department of Internal Medicine, Medical University of Graz, Graz, Austria

Recently the paradigm that the healthy lung is sterile was challenged and it is now believed that the lungs harbor a diverse microbiota also contributing to the pathogenesis of various diseases. Most of the research studies targeting the respiratory microbiome have focused on bacteria and their impact on lung health and lung diseases. Recently, also the mycobiome has gained attention. Lower respiratory tract (LRT) diseases (e.g., cystic fibrosis) and other diseases or conditions (e.g., HIV infection, lung transplantation, and treatment at intensive care units) have been investigated with regard to possible involvement of mycobiome in development or progression of diseases. It has been shown that diversities of mycobiome in the LRT vary in different populations and conditions. It has been proposed that the mycobiome diversity associated with LRT can vary with different stages of diseases. Overall, Candida was the dominant fungal genus in LRT samples. In this review, we summarize the recent findings regarding the human LRT mycobiome from a clinical perspective focussing on characterization of investigated patient groups and healthy controls as well as sampling techniques. From these data, clinical implications for further studies or routine practice are drawn. To obtain clinically relevant answers efforts should be enhanced to collect well characterized and described patient groups as well as healthy individuals for comparative data analysis and to apply thorough sampling techniques. We need to proceed with elucidation of the role of mycobiota in healthy LRT and LRT diseases to hopefully improve patient care.

Keywords: mycobiome, lower respiratory tract, Candida, cystic fibrosis, intensive care unit

\section{INTRODUCTION}

The lower respiratory tract (LRT) includes airways distal of the vocal cords, i.e., trachea, bronchi, bronchioles and anatomical structures of the lung, i.e., respiratory bronchioles, alveolar ducts, alveolar sacs, and alveoli (Schönbach, 2013). Fungal diseases affecting the LRT comprise invasive fungal infections (IFI) and diseases triggered by fungal LRT colonization or inhalation (e.g., allergic bronchopulmonary aspergillosis). Some respiratory diseases are possibly associated with fungal colonization, i.e., exacerbation of chronic obstructive pulmonary disease (COPD) or deterioration of lung function and structure in cystic fibrosis (CF) patients (Nguyen et al., 2015). Candida is often 
cultured from LRT samples but the clinical significance is still unknown. Baum (1960) started a discussion regarding the significance of Candida in the LRT by presenting data from conventional microbiological investigations of LRT samples. He described that the source of Candida present in the sputum samples was the mouth and that little significance could be attached to the finding of Candida within the sputum in the diagnosis of pulmonary candidiasis. Since then subsequent culture based studies investigating Candida in the LRT have given conflicting results (Corley et al., 1997; el-Ebiary et al., 1997; Eggimann et al., 2003; Meersseman et al., 2009).

In general, cultivation-independent molecular assays are more efficient in describing microbial communities than culturebased approaches which fail to detect the whole diversity of a microbiota and, therefore, hinder complete understanding of the interactions between the host and the microbiome (Ghannoum et al., 2010; Bittinger et al., 2014; Nguyen et al., 2015). However, compared to bacterial microbiota investigation surveys on the mycobiota of the LRT are still rare. Performing a literature search via PubMed 86 hits are generated by using "mycobiome" as a search term, 9 using "lung mycobiome" and 10 using "respiratory mycobiome" (four of the publications appear also in "lung mycobiome" search"; accessed November 21, 2016).

An overview of the current state of knowledge with respect to concepts of LRT mycobiota, the association of LRT mycobiota and fungal communities in other anatomical regions of the human body as well as the role of LRT mycobiota in some LRT diseases has been published recently (Nguyen et al., 2015). In comparison, the LRT mycobiota review presented here primarily addresses the characterization of investigated patient groups and healthy controls as well as utilized sampling techniques. From a clinical point of view, thorough selection of patient groups together with clear description of underlying diseases as well as antiinfective and non-antiinfective treatments that might influence bacterial and fungal microbiota are essential to detect relevant differences in fungal communities. From that informations it might be possible to draw clinically relevant conclusions and diagnostic as well as therapeutic implications. Beside differences in patient selection and molecular approaches, sampling techniques are also relevant for interpretation of results. In this review we therefore summarize recent literature addressing LRT mycobiome and its potential role in respiratory disease in certain patient groups and discuss the results from a clinical perspective including the potential influences by sampling techniques applied.

\section{ORAL MYCOBIOME}

Since LRT samples are commonly collected by procedures wherein the oral cavity is passed, the composition of the oral mycobiota is relevant to interpret LRT mycobiota studies. Although fungal colonization of the oral cavity is not imperatively

${ }^{1}$ http://www.ncbi.nlm.nih.gov/pubmed/?term=mycobiome connected to fungal colonization of other anatomical regions, e.g., the intestinal tract (Zollner-Schwetz et al., 2008), the contact of true LRT samples with saliva and/or mucosal surfaces occurring during expectoration of e.g., sputum samples might alter mycobiota composition in LRT samples. Oral mycobiota were investigated in healthy individuals to provide a basis for a detailed characterization of the oral mycobiome in health and disease. With results of that study the term mycobiome was introduced in medical literature as confirmed by our mycobiome literature search in PubMed starting with Ghannoum's paper published in 2010 (Ghannoum et al., 2010). In that study, oral rinse using $15 \mathrm{ml}$ of phosphate buffered saline was obtained from 20 healthy subjects (21-60 years of age, eight females and 12 males; non-smoking, no recent antifungal use, and no clinical signs of oral mucosal disease). Exclusion criteria were a history of receiving medication or treatment with topical or systemic steroids, pregnancy, and a history of insulin-dependent diabetes mellitus. Internal transcribed spacer (ITS) based sequencing revealed that the oral cavity contained 74 cultivable (most abundant genera: Candida, Cladosporium, Aureobasidium, Saccharomycetales) and 11 non-cultivable fungal genera. The low-abundance genera were considered to represent environmental fungi that have been transferred into the oral cavity by inhalation of spores or by ingesting fungal contaminated food (Ghannoum et al., 2010). As only healthy individuals were investigated the influence of antibacterial and antifungal therapy and certain immunosuppressive conditions predisposing for fungal infections (e.g., underlying diseases like hematooncological malignancies, diabetes mellitus, intensive care unit treatment, immunosuppressive treatments like corticosteroids, cyclophosphamide, antineoplastic chemotherapy, biological therapy) on oral mycobiota remain unknown. It should be mentioned, however, that this study lacked appropriate contamination controls and extraction blanks, thus the possible introduction of fungal signatures by the used reagents cannot be excluded.

\section{MYCOBIOME OF CYSTIC FIBROSIS PATIENTS}

In one of the first papers presenting LRT mycobiome data, eight sputum samples from four (two male and two female) adult CF patients (range 19-39 years) were investigated by pyrosequencing of ITS2 amplicons (Delhaes et al., 2012). For assessment of fungal and bacterial interaction $16 \mathrm{~S}$ rRNA gene sequencing was included. Two temporal sputum samples were collected from each clinically stable patient with a sampling interval of 1 year, but in one patient the sampling interval was only 3 months. Samples were collected by expectoration into a sterile cup after a water rinse. Two patients received azithromycin due to Pseudomonas colonization and other unspecified antibiotic therapies (two courses in one patient and up to seven courses in the second patient previous to the first sputum sample). Three patients had inhalative and one patient had additional systemic corticosteroid therapy. One patient was treated with 
itraconazole $600 \mathrm{mg}$ for 183 days due to bronchopulmonary allergic aspergillosis prior to sampling. Results showed high molecular diversities for the main fungal and bacterial taxa and high intra-individual differences as mycobiota changed between the sampling time points (e.g., 202 Candida reads in sample 1 and 8688 Candida reads in sample 2 in patient 1). Anaerobes were isolated together with Pseudomonas aeruginosa, and the latter was found more likely co-associated with Candida albicans than with Aspergillus fumigatus. Aside of the bacterial findings the authors concluded that the diversity and species richness of fungal communities was significantly lower in patients with decreased lung function and poor clinical status. It was further mentioned that C. albicans and C. parapsilosis, as part of the oral mycobiome, could migrate from the oral niche, and colonize and persist within the lower airways of CF patients. However, since Candida is the major genus found in the oral cavity as shown in the oral mycobiota study investigating healthy subjects mentioned above and since oral mycobiota have not been investigated in this CF study these conclusions must be interpreted with caution. It is not clear from that CF study whether "sputum" samples represented adequate sputum produced from the LRT and how the oral mycobiota impacted the LRT sputum mycobiota. In addition, the present CF cohort was small (four patients), included CF patients with different treatments that might affect LRT mycobiota (corticosteroids, antibiotics, and antimycotics), and lacked a specific control group. The association of reduced diversity and richness of fungal and bacterial microbiota with poor clinical status and lung function (reduced FEV1 and FVC) was mainly derived from two patients with preceding antibiotic therapy and antifungal therapy. Therefore, influence of antiinfective therapy on reduced microbial diversity and richness cannot be ruled out and the association with lung function remains questionable.

Another study encompassed six CF patients, which were admitted for antibiotic therapy due to respiratory disease exacerbation and Pseudomonas sp. positive sputum cultures. ITS based mycobiota analysis was performed with sputum samples collected from this cohort prior to and after systemic antibiotic treatment (Willger et al., 2014). One patient received tobramycin and meropenem; one tobramycin and ceftazidime; one tobramycin, ceftazidime and linezolid; two tobramycin, doripenem and vancomycin; and one tobramycin and doripenem. Inhalative antibacterial therapy (as applied in other CF cohorts as described below) was not mentioned in the studied patients as well as information on preceding antibacterial therapy was lacking. As CF patients repeatedly receive antibacterial (and sometimes antifungal) therapy for infectious exacerbation of underlying disease previous therapies might have impacted the observed LRT mycobiota results. In mycobiota analysis Candida represented the dominant genus with 11 different species (top three were C. albicans, C. dubliniensis, and C. parapsilosis) and accounted for 74-99.9\% of all ITS1 reads in all samples making these species both the most abundant and also the most prevalent. However, there was a broad distribution of $C$. albicans relative abundance ranging from $0.1 \%$ of reads (post-treatment sample from subject number 2) to $98.8 \%$ of reads (post-treatment sample from subject number 6). Besides C. albicans only Malassezia species occurred in all samples with 10- to 50-fold lower reads compared to Candida species. Also non-fumigatus Aspergillus species were detected at low levels. The relative abundance of Candida species was stable during antibiotic treatment and antibiotic treatment did not establish a specific fungal community. Fungal density varied after completion of antibiotic treatment with increase or decrease in individual patients but no common trend could be observed. Overall, no specific trend in fungal burden associated with antibacterial therapy could be detected. In contrast to decreased bacterial richness after antibacterial treatment no significant difference was determined for fungal richness although a trend for a decrease was observed. Candida and Malassezia species accounted for more than $80 \%$ of all assigned reads per sample and persisted throughout antibacterial treatment without significant change in relative abundance suggesting stability within the mycobiome. As with the other CF study, it is not clear if "sputum" samples represented adequate sputum produced from the LRT and if the oral mycobiota contaminated and impacted presumed LRT sputum mycobiota. For further clarification of the role of mycobiota in LRT the authors also suggested methods to differentiate between dead or alive microorganisms to assess active microbiota in the LRT.

In another study 72 sputum samples were collected from $56 \mathrm{CF}$ patients recruited in an ambulatory center including a subcohort of 13 patients who provided sputum two (10 patients) or three (three patients) times within a 2-years sampling period (Kramer et al., 2015a). Sputum samples were collected in duplicate, stored at $-20^{\circ} \mathrm{C}$ and subsequently analyzed by ITS primer based PCR, preparation of single stranded DNA, single-strand conformation polymorphism electrophoresis and sequencing of individual bands. C. albicans and $C$. dubliniensis were the most dominant fungal species in the CF cohort, with $44.4 \%$ and $23.6 \%$ positive samples, respectively, followed by Saccharomyces cerevisiae, with $19.4 \%$, and C. parapsilosis, with $13.8 \%$ positive samples. Several other fungi were detected at lower rates including Aspergillus sp., and Cladosporium sp., Scedosporium and Exophiala sp. In patients investigated at two time points different fungi were detected in the same patient indicating dynamic mycobiota composition. In concordance with the other CF study mentioned above it is not clear from this particular CF study if "sputum" samples represented adequate sputum produced from the LRT and how oral mycobiota impacted presumed LRT sputum mycobiota (as sputum passes the oral cavity). In another study published by the same group the antibiotic therapy of the same CF cohort was described in more detail. Investigated CF patients were under continuous treatment of antibiotics by inhalation applied twice a day at home (mostly colistin or tobramycin according to clinical needs). Interestingly, none of the patients received antibiotics intravenously during the 2-years time frame of study (Kramer et al., 2015b). Information on antimycotic therapy during or prior to sampling was not provided. Even if the CF cohort 
represented routine clinical cases conclusions from mycobiota (and microbiota) analysis can hardly be drawn based on the missing information regarding antifungal therapy and the incomplete description of inhalative antibiotic therapy (how many patients received which inhalative antibacterial medication?).

\section{MYCOBIOME OF PATIENTS WITH ASTHMA BRONCHIALE}

Lower respiratory tract mycobiota were investigated in 30 asthma patients and compared to 13 controls (van Woerden et al., 2011, 2013). Patients were free of oral steroid use $\geq 2$ weeks prior to induced sputum sampling but most of them were on inhaled corticosteroids. No information regarding previous or current antibiotic or antifungal therapy was provided. After nebulizing isotonic saline induced sputum was coughed into petri dishes and spread on microscopic slides for microscopic examination. Afterward a $5 \mathrm{~mm}^{2}$ area was subsequently excised from the slide for DNA extraction and PCR. The excised samples from each study group were combined to yield two pooled samples, one from all asthma patient sputum samples and one from all healthy control subject sputum samples. Unfortunately, as described by the authors, a sample from one asthma patient was inadvertently included in the control set. Primer pairs Euk1a and Euk516r were used for PCR, sequenced using a 454 pyrosequencer and DNA sequences compared to SILVA database (van Woerden et al., 2013). In total 136 fungal species were identified, with Psathyrella and Malassezia spp. representing each approximately $25 \%$ of all reads in asthma patients and Eremothecium spp. representing approximately $40 \%$ of all reads in healthy controls. As the authors noted in their discussion pooling sputum samples prevented clear interpretation of results as fungi identified could origin from one individual heavily colonized or from several individuals colonized with identical or nearly similar fungal species. In addition, oral mycobiota as well as material used for sampling (petri dishes, slides, and saline) and its impact on mycobiota results by potential contamination was not investigated. Considering fungal species found in asthma patients the pathophysiological role of Psathyrella candolleana, a mushroom found on lawns or pastures in Europe and North America, is unknown as well as the role of Eremothecium sinecaudum, a yeast with pathogenic properties for plants, in healthy subjects. The duration for collection of pooled sputum and time intervals between sample collection and storage was not provided as well as storing conditions. It has been shown that prolonged time between sample collection and sample storage (more than $12 \mathrm{~h}$ ) as well as more than four freeze thaw cycles distorted microbiota profiles (Cuthbertson et al., 2014, 2015). As mentioned above, fungi found in sputum from asthma patients and healthy controls mainly contained environmental fungi present in plants and grassland (van Woerden et al., 2013). Thus contamination of the pooled sputum from both study groups during sampling and storage cannot be ruled out. As sputum samples were mixed up from individual patients and the pooled control sample included one asthma sample interpretation of results and considerations of differences between study populations is very limited.

\section{MYCOBIOME OF PATIENTS WITH LUNG TRANSPLANTS}

Mycobiota based on ITS-sequencing of 21 lung transplant patients were investigated in BAL and oropharyngeal washes and compared to healthy controls, the latter undergoing a two-bronchoscope sampling technique or conventional bronchoscopy (number of healthy controls undergoing each of the sampling techniques was not provided in the text; in figures within the manuscript data from six healthy controls were shown). Two patients undergoing routine bronchoscopy for evaluation of sarcoidosis and a lung nodule representing adenocarcinoma (but both without lung transplantation) were also included, but they were not further characterized (Charlson et al., 2012). All lung transplant patients had immunosuppressive and antibiotic therapy, three received voriconazole and six nystatin (one patient had both antifungals). Prior to bronchoscopy instruments were flushed with $10 \mathrm{ml}$ saline to investigate microbial DNA present in bronchoscopes and saline. Fungal populations in lung transplant patients were typically dominated by Candida in oral washes and BAL and by Aspergillus in BAL but not in oral washes. Oral washes of lung transplant patients revealed 13 samples with high fungal sequences, all but one were dominated by Candida. Patients without Candida in BAL had no (six patients) or low (two patients) Candida sequences in oral washes. As eight lung transplant patients had nystatin and/or voriconazole therapy the presence or absence of Candida and other fungi (targeted by nystatin and voriconazole) in oral washes and BAL is hard to assess. Nystatin did not consistently decrease or eliminate Candida from oral washes but patients on voriconazole had no Candida sequences in BAL (patient no. 34 was only presented in Figure E2, supplementary material of the article; Charlson et al., 2012). However, in two of voriconazole treated patients Aspergillus sequences were detected (patient no. 34, also with positive Aspergillus culture, and patient no. 43). One patient (no. 36) had Aspergillus in culture and in mycobiota analysis but in a second BAL 2 months later Aspergillus was no longer detected by both methods while the patient was treated with voriconazole; voriconazole obviously eliminated Aspergillus in that particular patient. Candida was absent in BAL of healthy volunteers although Candida was present in oral washes. Unfortunately no information regarding a potential pathogenetic role of fungi detected within mycobiota was provided for the presented lung transplant patients. The presence or absence of invasive fungal infection or fungal breakthrough infection in voriconazole treated patients (based on clinical data, radiological examination, biomarkers like galactomannan or 1,3-beta-Dglucan testing, voriconazole trough level) and outcome was not mentioned. 


\section{MYCOBIOTA IN MISCELLANEOUS PULMONARY DISEASES AND HIV POSITIVE PATIENTS}

In a study published by the same authors as mentioned above in the lung transplant patients, data from an almost similar study cohort included mycobiota from 42 lung transplant patients, 19 HIV positive patients, 13 patients with various pulmonary diseases and 12 healthy controls (Bittinger et al., 2014). Four control patients were counted twice as they were investigated by a double bronchoscopy and single bronchoscopy technique at least 1 year apart. Healthy and HIV positive subjects were described not to have respiratory symptoms and antibiotic therapy. However, in the Table 1 one HIV patient did in fact have antibiotic therapy (clindamycin within 3 months prior to sampling) and another one had inhalative corticosteroid treatment. Subjects within the healthy and HIV positive study groups where smokers and non-smokers. In patients with various pulmonary diseases two patients had immunosuppressive therapy, two had systemic antibiotic therapy but two patients with pneumonia surprisingly had no antibiotic therapy. Two out of 24 lung transplant patients refused collection of clinical data. Thirteen of the remaining 22 lung transplant patients had antifungal therapy (11 nystatin, two voriconazole treatments). Twenty received antibiotic therapy (mainly trimethoprim and sulfamethoxazole), two had atovaquone therapy and all received immunosuppressants (presented in Supplementary Table S3 of Bittinger et al., 2014). The authors also focused on analysis of possible confounding factors as 132 samples from laboratory water, bronchoscope canal flushes, saline used for oral washes and BAL, sterile swabs and lab tabletop surface were investigated [supplemental report in (Bittinger et al., 2014)]. Interestingly, the composition of fungi in BAL samples was generally similar to that in contamination controls. After correction of abundance by subtraction of reads found in the control samples (e.g., saline) Candida was mainly found in BAL and oral washes of the study groups (Figure 37-43 in supplemental report of Bittinger et al., 2014) as well as Aspergillus and Cladosporium in lung transplant patients and those with miscellaneous pulmonary diseases (Figure 37 and 38 in supplementary report of Bittinger et al., 2014). Clinical significance of fungal detection in presented patient groups is unclear from that study as clinical data including radiological examination, biomarkers, introduction of antifungal treatment and outcome have not been reported.

Another study provided mycobiota data from $32 \mathrm{HIV}$ positive (10 with COPD) and $24 \mathrm{HIV}$-uninfected patients extracted from a cohort comprising $396 \mathrm{HIV}$-infected and HIV-uninfected individuals with smokers and marijuana abusers in both study groups (Cui et al., 2015). Prior to sampling study subjects had stable respiratory symptoms in previous 4 weeks and did not receive antibiotic or immunosuppressive therapy in previous 6 months. Oral wash, induced sputum $(<30 \%$ squamous cell count) and BAL passing the oral cavity were collected

TABLE 1 | Overview of studies investigating LRT mycobiota.

\begin{tabular}{|c|c|c|c|}
\hline $\begin{array}{l}\text { Study author, year of } \\
\text { publication }\end{array}$ & $\begin{array}{l}\text { Patients (pts)/study subjects } \\
\text { (controls) }\end{array}$ & Sampling method & Material analyzed \\
\hline Delhaes et al., 2012 & four CF pts & sputum expectoration & sputum \\
\hline Willger et al., 2014 & six CF pts & sputum expectoration & sputum \\
\hline Kramer et al., 2015a & 56 CF pts & sputum expectoration & sputum \\
\hline $\begin{array}{l}\text { van Woerden et al., } 2013 \\
\text { van Woerden et al., } 2011\end{array}$ & $\begin{array}{l}30 \text { asthma bronchiale pts } \\
13 \text { controls }\end{array}$ & induced sputum expectoration & $\begin{array}{l}\text { two pooled sputum samples (one from } \\
\text { patients and one from controls) }\end{array}$ \\
\hline \multirow[t]{2}{*}{ Charlson et al., 2012} & $\begin{array}{l}21 \text { lung transplant pts } \\
\text { one patient with suspected sarcoidosis } \\
\text { one patient with adenocarcinoma }\end{array}$ & $\begin{array}{l}\text { bronchoscopy (including 2-bronchoscope } \\
\text { sampling in healthy controls) } \\
\text { oral washes with saline } \\
\text { bronchoscope saline flush }\end{array}$ & $\begin{array}{l}\text { BAL } \\
\text { Oral washes } \\
\text { saline and bronchoscope flush }\end{array}$ \\
\hline & six controls & & \\
\hline Bittinger et al., 2014 & $\begin{array}{l}42 \text { lung transplant pts } \\
19 \text { HIV pts } \\
13 \text { pts with various pulmonary diseases } \\
12 \text { controls }\end{array}$ & $\begin{array}{l}\text { bronchoscopy (including 2-bronchoscope } \\
\text { sampling in healthy controls) } \\
\text { oral washes with saline } \\
\text { bronchoscope saline flush } \\
\text { swabs }\end{array}$ & $\begin{array}{l}\text { BAL } \\
\text { oral washes } \\
\text { laboratory water } \\
\text { bronchoscope flushes } \\
\text { saline } \\
\text { sterile swabs } \\
\text { lab tabletop surface }\end{array}$ \\
\hline Cui et al., 2015 & $\begin{array}{l}32 \text { HIV pts [10 with chronic obstructive } \\
\text { pulmonary disease (COPD)] } \\
24 \text { HIV negative pts }\end{array}$ & $\begin{array}{l}\text { oral washes with saline } \\
\text { induced sputum } \\
\text { bronchoscopy }\end{array}$ & $\begin{array}{l}\text { oral washes } \\
\text { sputum } \\
\text { BAL }\end{array}$ \\
\hline Bousbia et al., 2012 & $\begin{array}{l}160 \text { ICU pts with pneumonia } \\
25 \text { ICU pts without pneumonia }\end{array}$ & bronchoscopy & $\mathrm{BAL}$ \\
\hline Krause et al., 2016 & $\begin{array}{l}39 \text { ICU pts } \\
\text { seven non-ICU patients with antibiotic } \\
\text { therapy } \\
\text { five ICU pts for sampling comparison } \\
\text { eight controls }\end{array}$ & $\begin{array}{l}\text { bronchoscopy through endotracheal tubes } \\
\text { suction of endobronchial secretion through } \\
\text { endotracheal tubes } \\
\text { bronchoscope saline flush }\end{array}$ & $\begin{array}{l}\text { BAL } \\
\text { endobronchial secretion } \\
\text { bronchoscope flushes } \\
\text { saline }\end{array}$ \\
\hline
\end{tabular}


as well as control samples by aspirating saline through the bronchoscope channel. Fungal microbiota were analyzed by sequencing amplicons generated by PCR using ITS- and 18Stargeting primers. $18 \mathrm{~S}$ data were used for operational taxonomic unit (OTU)-type analysis and ITS data were used for specieslevel analysis. The authors mentioned that the 18S rRNA gene gave higher PCR-positive rates, but fewer fungal reads compared with the ITS, because the $18 \mathrm{~S}$ primers cross-reacted with host (=human); consequently, approximately $90 \%$ of the raw $18 \mathrm{~S}$ sequencing reads were human. By using $18 \mathrm{~S}$ data for comparison of different sampling techniques, the authors interestingly found that induced sputum shared little similarity with BAL. In principle coordinate analysis of $18 \mathrm{~S}$ data of healthy subjects (HIV negative, normal lung function) using only $30 \%$ of all data, BAL samples clustered together with oral wash, induced sputum overlapped in part with oral wash samples but not with BAL. A similar distribution pattern was seen with the entire cohort including HIV-infected and HIV-uninfected individuals with or without normal lung function. Candida represented more than $90 \%$ of the ITS reads and was clearly dominant in oral washes compared with BAL. There was also a higher prevalence of Candida in induced sputum compared with BAL. Ceriporia lacerata, Saccharomyces cerevisiae, and Penicillium brevicompactum were predominant in BAL compared to oral wash. None of these species were detected in the corresponding bronchoscopic control samples, indicating that environmental contamination was unlikely. In HIV infected individuals nine species including Pneumocystis jirovecii (confirmed by nested PCR) were overrepresented in BAL compared to HIV-uninfected individuals. No species was associated with COPD in the HIVinfected cohort as compared with HIV-infected individuals with normal lung function. In marijuana smokers, with and without $\mathrm{HIV}$ infection, four species (including two that are known plant pathogens, Phialocephala virens and Taphrina tormentillae) were overrepresented as compared with individuals who had not smoked marijuana in the year prior to sampling. The authors mentioned that smoking marijuana without filters might have contributed to inhalation of this certain environmental/plant fungi. Considering all subjects investigated, fungal communities in the LRT resembled those in the mouth. This result could be caused by microaspiration of oral content, contamination during bronchoscopy when passing the oral cavity, contamination of induced sputum with oral microbiota or contamination of oral microbiota by coughing or exhalation. The latter is unlikely as oral wash was collected prior to sputum sampling or bronchoscopy.

\section{MYCOBIOME IN LRT OF ICU PATIENTS}

Mycobiota in LRT of ICU patients were investigated in two studies. In the first study $18 \mathrm{~S}$ rRNA gene sequencing was performed to analyze the etiology of pneumonia in ICU patients with community acquired pneumonia (CAP, 32 episodes), ventilator-associated pneumonia (VAP, 106 episodes), nonventilator ICU pneumonia (NV-ICU P, 22 episodes), aspiration pneumonia (AP, 25 episodes) and compared to $25 \mathrm{ICU}$ patients without pneumonia (CS; Bousbia et al., 2012). As pneumonia cases were counted as episodes (185 pneumonia episodes) the total number of patients included in each of the pneumonia subgroups is not clear. However, in some tables the number of pneumonia patients were 185 enabling the conclusion that one pneumonia episode obviously corresponded to one patient. Clinical data regarding underlying diseases beside pneumonia, (previous) antibiotic and antimycotic therapy or initiation of new antiinfective therapy, presence or absence of tracheal tubes, invasive or non-invasive mechanical ventilation and underlying diseases were not provided. Detailed sampling procedures, e.g., time of BAL sampling after admission or diagnosis or pneumonia, BAL by bronchoscopy passing the oral cavity or through oropharyngeal ventilation tubes, transport and storage of BAL cultures, investigation of material used for $\mathrm{BAL}$, investigation of pre-BAL bronchoscopy flushes were not mentioned. Interestingly, ICU patients used as control patients (=no pneumonia) also had ARDS but with comparable or even higher numbers compared to some pneumonia patients (38\% in CAP, 33\% in VAP, $41 \%$ in NV-ICU P, $12 \%$ in AP; $31 \%$ in all pneumonia patients and $28 \%$ in patients without pneumonia considered as controls). The proportion of patients with immunosuppressive therapy in each pneumonia group was reported but the type of immunosuppressive therapy was not provided (38\% in CAP, $40 \%$ in VAP, $50 \%$ in NV-ICU P, 36\% in AP; $40 \%$ in all pneumonia patients and $16 \%$ in controls). C. albicans signatures were most abundant in all patients groups and controls. C. utilis showed higher abundance in controls compared to pneumonia subgroups ( $p=0.01$; Supplementary Table S5 in supplementary material of Bousbia et al., 2012). Aspergillus, Penicillium and Cladophialophora genera were dominant in the CAP cohort but did not reach significant differences compared to other cohorts. Tremellomycetes (represented by Cryptococcus) was identified in NV-ICU P, whereas Agaricomycetes and unclassified Ascomycota were only identified in VAP patients. Sordariomycetes were only identified in controls. However, maybe based on small numbers, no significant difference could be calculated for these classes. Beside bacterial and fungal microbiome analysis the authors included a lot of other microbiological methods to detect microorganisms causing pneumonia. For many of detected microorganisms including those found in mycobiota analysis the authors raised the question on the actual role of these microorganisms in pneumonia. The authors concluded that the respiratory microbiota was more complex than expected (Bousbia et al., 2012).

In another study, LRT mycobiota in non-neutropenic intubated and mechanically ventilated ICU patients with antibiotic therapy to treat pneumonia were investigated and compared to several other groups: healthy controls without preceding antibiotic therapy, patients without pulmonary diseases with antibiotic therapy, non-neutropenic intubated and mechanically ventilated ICU patients without preceding or current antibiotic therapy, non-neutropenic intubated and mechanically ventilated ICU patients without pulmonary disease but with antibiotic therapy for treatment of extrapulmonary infection. Smoking behavior of study patients was not evaluated. All study subjects were without antimycotic treatment $\geq 8$ weeks 
prior to sampling (Krause et al., 2016). LRT in all patients and healthy study subjects were sampled through endotracheal tubes avoiding contact to the oral cavity. Non-ICU patients and healthy controls were sampled by aspiration of endobronchial secretion and ICU patients were sampled by bronchoscopic guided BAL. Saline used for BAL and collected after flushing the sampling channel of the bronchoscope immediately before bronchoscopy was also investigated. Sampling techniques (endobronchial secretion versus BAL) showed similar results and saline did not influence mycobiota results. The results showed that Candida was part of the fungal microbiota of various intubated and mechanically ventilated ICU patients with and without antibiotic therapy and with and without pneumonia (59-73\% Candida signatures within 5-8 fungal genera; Krause et al., 2016). Candida sequences were not present in mycobiota of non-ICU patients with antibiotic therapy and healthy controls. Admission to and treatment on ICUs shifted LRT fungal microbiota to Candida spp. dominated fungal profiles but antibiotic therapy did not. Mycobiota analysis was not done with samples from the oral cavity but in conventional cultures antibiotic therapy increased the prevalence of Candida spp. in this compartment. Conventional cultures also showed that admission to the ICU increased the prevalence of Candida spp. in the LRT, but other fungi were not detected by culture. None of the ICU patients had candidemia or other invasive fungal disease as determined by clinical, imaging, laboratory and microbiological investigations.

\section{DISCUSSION}

The mycobiota in the LRT is diverse and varies in different populations and LRT diseases. Links to certain stages of diseases or LRT dysfunction have been proposed. In one study the diversity and species richness of fungal and bacterial communities were significantly lower in patients with decreased lung function as assessed by forced expiratory pressure in $1 \mathrm{~s}$ in lung function tests (Delhaes et al., 2012). However, the association was mainly derived from two patients with preceding antibiotic therapy and antifungal therapy and the contribution of fungal or bacterial microbiota to lung function could not be distinguished. Despite variable fungal composition in LRT, Candida overall dominated mycobiota in most of the studies including CF patients, lung transplant patients, HIV infected patients and ICU patients (Bousbia et al., 2012; Charlson et al., 2012; Bittinger et al., 2014; Willger et al., 2014; Cui et al., 2015; Kramer et al., 2015a; Krause et al., 2016). In remaining studies with dominating fungi other than Candida methodologies were too weak to draw profound conclusions. However, Candida was also the most abundant genus in oral mycobiome analysis (Ghannoum et al., 2010) questioning the results of studies that presented sputum samples without quality control (e.g., $<10$ squamous cells in high power field sputum examination). Unfortunately, even in bronchoscopy guided BAL contact to oral mucosa or saliva during insertion of the bronchoscope might alter microbiota composition of BAL obtained through orally contaminated bronchoscopy tips. But LRT mycobiota similar or close to oral mycobiota does not necessarily represent contamination of bronchoscopes or translocation of mycobiota by bronchoscopy, as upper and LRT might be colonized by identical or closely related microorganisms. Similar or close mycobiota in upper and LRT analysis could also be caused by microaspiration of oral content, contamination of induced sputum with oral microbiota or contamination of oral microbiota by coughing or exhalation. To overcome limitations in interpreting mycobiota data sampling techniques bypassing the oral cavity (e.g., via endotracheal tubes) or two-bronchoscopy sampling techniques should therefore be preferred to obtain true LRT samples. By applying such techniques and therefore exclusion of contamination, mycobiota in upper and LRT could be assessed more reliable.

In two previous studies reagents (e.g., saline, lab water, lab surfaces) have been analyzed to assess its influence on microbiota results from BAL (Bittinger et al., 2014; Krause et al., 2016); however, in other studies this was not done (Erb-Downward et al., 2011; van Woerden et al., 2011, 2013). Nevertheless, none of the studies investigating reagents showed that Candida abundance reached $>50 \%$ as has been found in BAL samples from ICU patients (Krause et al., 2016). The reason for the elevated Candida abundance in LRT of ICU patients is not clear. The role of ICU environment or translocation of microorganisms from intestinal mycobiota (via ICU personnel or retrograde translocation up to the oral cavity followed by microaspiration passing the cuff or the orotracheal tube) in elevated LRT Candida abundance or in shifts of LRT mycobiota has not been investigated yet.

Three studies included sequential sampling for assessment of dynamics in mycobiota composition over time. Observation periods varied between days (ICU patients; Krause et al., 2016), weeks (CF patients; Willger et al., 2014), 3 months to 1 year (CF patients; Delhaes et al., 2012), and months up to 2 years (CF patients; Kramer et al., 2015a). Conclusions are difficult to draw based on low numbers of individuals investigated, different patient groups and differences in time frames elapsing between sampling. Whereas high intraindividual differences with changes in Candida abundance were found in one CF study (Delhaes et al., 2012) another CF study found stable results (Willger et al., 2014). The third CF study found differences in number of fungal genera in some patients providing two sputum samples (Kramer et al., 2015a). In ICU patients fungal microbiota were stable in 3 of 4 patients as shown by analysis of first and second BAL sample 4 to 7 days apart (Krause et al., 2016). Two studies compared different LRT sampling techniques in individual patients, i.e., induced sputum and BAL in one study (Cui et al., 2015) and endotracheal aspiration and BAL in another study (Krause et al., 2016). Whereas differences were detected between sputum and BAL based on $18 \mathrm{~S}$ rRNA sequencing no difference was found between endotracheal aspirate and BAL based on ITS sequencing. As the authors mentioned in $18 \mathrm{~S}$ based sequencing human $18 \mathrm{~S}$ rRNA reads reduced the sequencing depth of fungal $18 \mathrm{~S}$ rRNA gene reads, which might have influenced comparative data analysis of sputum and BAL. Although some studies included conventional cultures for assessment of microbial composition all studies mentioned above did not account for viability of microbiota explored by sequencing methods. Therefore it is almost unknown if detected mycobiota represent true alive residential or inhaled dead microorganisms or both. Future studies need to include this 
aspect by e.g., dividing samples and treating of one half with propidium monoazide (PMA) to select for dead cells (Nocker et al., 2007).

The role of Candida in LRT in above mentioned diseases and conditions is still largely unknown. Patient characteristics provided in some mycobiota studies prohibit clinical conclusions as relevant data regarding underlying diseases, presence or absence of LRT infectious disease, initiation of antibiotic or antimycotic therapy, and outcome (e.g., invasive fungal infection) are missing. In the study investigating asthma bronchiale patients no information regarding previous or current antibiotic or antifungal therapy was provided (van Woerden et al., 2013). As shown in one of the studies in ICU patients (Krause et al., 2016) antibiotic therapy surprisingly did not influence mycobiota. Interestingly, antibiotic therapy in CF patients overall also had no impact on mycobiota composition and fungal burden although fungal burden varied between individual patients with decreasing and increasing levels (Willger et al., 2014). The influence of antimycotic therapy on LRT mycobiota is still unclear but some antimycotics might alter LRT mycobiota as detected in one patient in the lung transplant patient group discussed above (Charlson et al., 2012).

\section{CONCLUSION}

Although some studies suggest renewal of our pathophysiological understanding of mycobiota in LRT, shortcomings in clinically relevant methodologies in some studies call for further investigations to compensate previous deficiencies. Improved study settings will allow to draw better clinically relevant conclusions regarding diagnostic or therapeutic procedures in certain LRT diseases. As previously suggested, future mycobiota

\section{REFERENCES}

Baum, G. L. (1960). The significance of Candida albicans in human sputum. N. Engl. J. Med. 263, 70-73. doi: 10.1056/NEJM1960071426 30204

Bittinger, K., Charlson, E. S., Loy, E., Shirley, D. J., Haas, A. R., Laughlin, A., et al. (2014). Improved characterization of medically relevant fungi in the human respiratory tract using next-generation sequencing. Genome Biol. 15:487. doi: 10.1186/PREACCEPT-1218610333125217

Bousbia, S., Papazian, L., Saux, P., Forel, J. M., Auffray, J.-P., Martin, C., et al. (2012). Repertoire of intensive care unit pneumonia microbiota. PLoS ONE 7:e32486. doi: 10.1371/journal.pone.0032486

Charlson, E. S., Diamond, J. M., Bittinger, K., Fitzgerald, A. S., Yadav, A., Haas, A. R., et al. (2012). Lung-enriched organisms and aberrant bacterial and fungal respiratory microbiota after lung transplant. Am. J. Respir. Crit. Care Med. 186, 536-545. doi: 10.1164/rccm.201204-0693OC

Corley, D. E., Kirtland, S. H., Winterbauer, R. H., Hammar, S. P., Dail, D. H., Bauermeister, D. E., et al. (1997). Reproducibility of the histologic diagnosis of pneumonia among a panel of four pathologists: analysis of a gold standard. Chest 112, 458-465. doi: 10.1378/chest.112.2.458

Cui, L., Lucht, L., Tipton, L., Rogers, M. B., Fitch, A., Kessinger, C., et al. (2015). Topographic diversity of the respiratory tract mycobiome and alteration in HIV and lung disease. Am. J. Respir. Crit. Care Med. 191, 932-942. doi: 10.1164/ rccm.201409-1583OC

Cuthbertson, L., Rogers, G. B., Walker, A. W., Oliver, A., Hafiz, T., Hoffman, L. R., et al. (2014). Time between collection and storage significantly influences bacterial sequence composition in sputum samples from cystic fibrosis (and bacterial microbiota) studies should enable to answer one or more of the following questions: Can experiments detect differences that matter (in the context of disease and treatment)? Does the study show causation or just correlation? What is the mechanism? How much do experiments reflect reality? Could anything else explain the results? (Hanage, 2014). To obtain clinically relevant answers efforts should be enhanced to collect well characterized and described patient groups as well as healthy individuals for comparative data analysis and to apply thorough sampling techniques. For clinical routine we learn from current stage of knowledge that conventional microbiology obviously does not reflect true composition of LRT mycobiome. However, therapeutic studies based on new sequencing techniques are missing. We need to proceed with elucidation of the role of mycobiota in healthy LRT and LRT diseases to hopefully improve patient care. This might include diagnostic procedures with cultivation-independent molecular assays and prophylactic as well as therapeutic interventions in patients potentially suffering from mycobiota alterations (e.g., CF patients, lung transplant patients, ICU patients).

\section{AUTHOR CONTRIBUTIONS}

All authors listed, have made substantial, direct and intellectual contribution to the work, and approved it for publication.

\section{ACKNOWLEDGMENTS}

This work was supported by the Austrian Science Fund, grant number P 23037-B18 and KLI 561-B31.

respiratory infections. J. Clin. Microbiol. 52, 3011-3016. doi: 10.1128/JCM. 00764-14

Cuthbertson, L., Rogers, G. B., Walker, A. W., Oliver, A., Hoffman, L. R., Carroll, M. P., et al. (2015). Implications of multiple freeze-thawing on respiratory samples for culture-independent analyses. J. Cyst. Fibros. 14, 464-467. doi: 10.1016/j.jcf.2014.10.004

Delhaes, L., Monchy, S., Fréalle, E., Hubans, C., Salleron, J., Leroy, S., et al. (2012). The airway microbiota in cystic fibrosis: a complex fungal and bacterial community-implications for therapeutic management. PLoS ONE 7:e36313. doi: 10.1371/journal.pone.0036313

Eggimann, P., Garbino, J., and Pittet, D. (2003). Epidemiology of Candida species infections in critically ill non-immunosuppressed patients. Lancet Infect. Dis. 3, 685-702. doi: 10.1016/S1473-3099(03)00801-6

el-Ebiary, M., Torres, A., Fàbregas, N., la Bellacasa, J. P., González, J., Ramirez, J., et al. (1997). Significance of the isolation of Candida species from respiratory samples in critically ill, non-neutropenic patients. An immediate postmortem histologic study. Am. J. Respir. Crit. Care Med. 156, 583-590. doi: 10.1164/ ajrccm.156.2.9612023

Erb-Downward, J. R., Thompson, D. L., Han, M. K., Freeman, C. M., McCloskey, L., Schmidt, L. A., et al. (2011). Analysis of the lung microbiome in the "healthy" smoker and in COPD. PLoS ONE 6:e16384. doi: 10.1371/journal.pone.0016384

Ghannoum, M. A., Jurevic, R. J., Mukherjee, P. K., Cui, F., Sikaroodi, M., Naqvi, A., et al. (2010). Characterization of the Oral Fungal Microbiome (Mycobiome) in Healthy Individuals. PLoS Pathog. 6:e1000713. doi: 10.1371/journal.ppat. 1000713

Hanage, W. P. (2014). Microbiology: microbiome science needs a healthy dose of scepticism. Nature 512, 247-248. doi: 10.1038/512247a 
Kramer, R., Sauer-Heilborn, A., Welte, T., Guzman, C. A., Abraham, W.-R., and Höfle, M. G. (2015a). Cohort study of airway mycobiome in adult cystic fibrosis patients: differences in community structure between fungi and bacteria reveal predominance of transient fungal elements. J. Clin. Microbiol. 53, 2900-2907. doi: 10.1128/JCM.01094-15

Kramer, R., Sauer-Heilborn, A., Welte, T., Jauregui, R., Brettar, I., Guzman, C. A., et al. (2015b). High individuality of respiratory bacterial communities in a large cohort of adult cystic fibrosis patients under continuous antibiotic treatment. PLOS ONE 10:e0117436. doi: 10.1371/journal.pone.011 7436

Krause, R., Halwachs, B., Thallinger, G. G., Klymiuk, I., Gorkiewicz, G., Hoenigl, M., et al. (2016). Characterisation of Candida within the mycobiome/microbiome of the lower respiratory tract of ICU patients. PLoS ONE 11:e0155033. doi: 10.1371/journal.pone.0155033

Meersseman, W., Lagrou, K., Spriet, I., Maertens, J., Verbeken, E., Peetermans, W. E., et al. (2009). Significance of the isolation of Candida species from airway samples in critically ill patients: a prospective, autopsy study. Intensive Care Med. 35, 1526-1531. doi: 10.1007/s00134-009-1482-8

Nguyen, L., Viscogliosi, E., and Delhaes, L. (2015). The lung mycobiome: an emerging field of the human respiratory microbiome. Front. Microbiol. 6:89. doi: $10.3389 /$ fmicb. 2015.00089

Nocker, A., Sossa-Fernandez, P., Burr, M. D., and Camper, A. K. (2007). Use of propidium monoazide for live/dead distinction in microbial ecology. Appl. Environ. Microbiol. 73, 5111-5117. doi: 10.1128/AEM.02987-06

Schönbach, C. (2013). "Respiratory tract, upper and lower," in Encyclopedia of Systems Biology, edsW. Dubitzky, O. Wolkenhauer, K.-H. Cho, and H. Yokota (New York, NY: Springer), 1851-1852. doi: 10.1007/978-1-4419-98637_748 van Woerden, H. C., Gregory, C., Brown, R., Marchesi, J. R., Hoogendoorn, B., and Matthews, I. P. (2013). Differences in fungi present in induced sputum samples from asthma patients and non-atopic controls: a community based case control study. BMC Infect. Dis. 13:69. doi: 10.1186/1471-2334-13-69

van Woerden, H. C., Ratier-Cruz, A., Aleshinloye, O. B., Martinez-Giron, R., Gregory, C., and Matthews, I. P. (2011). Association between protozoa in sputum and asthma: a case-control study. Respir. Med. 105, 877-884. doi: 10. 1016/j.rmed.2010.11.016

Willger, S. D., Grim, S. L., Dolben, E. L., Shipunova, A., Hampton, T. H., Morrison, H. G., et al. (2014). Characterization and quantification of the fungal microbiome in serial samples from individuals with cystic fibrosis. Microbiome 2:40. doi: 10.1186/2049-2618-2-40

Zollner-Schwetz, I., Auner, H. W., Paulitsch, A., Buzina, W., Staber, P. B., Ofner-Kopeinig, P., et al. (2008). Oral and intestinal Candida colonization in patients undergoing hematopoietic stem-cell transplantation. J. Infect. Dis. 198, 150-153. doi: 10.1086/588827

Conflict of Interest Statement: The authors declare that the research was conducted in the absence of any commercial or financial relationships that could be construed as a potential conflict of interest.

Copyright (C) 2017 Krause, Moissl-Eichinger, Halwachs, Gorkiewicz, Berg, Valentin, Prattes, Högenauer and Zollner-Schwetz. This is an open-access article distributed under the terms of the Creative Commons Attribution License (CC BY). The use, distribution or reproduction in other forums is permitted, provided the original author(s) or licensor are credited and that the original publication in this journal is cited, in accordance with accepted academic practice. No use, distribution or reproduction is permitted which does not comply with these terms. 